\title{
Prompt Ultraviolet-to-Soft-X-Ray Emission of Gamma-Ray Bursts: Application to GRB 031203?
}

\author{
Zhuo $\operatorname{Li}^{1}$ and L. M. Song ${ }^{1}$
}

\begin{abstract}
We discuss the prompt emission of GRBs, allowing for $\gamma \gamma$ pair production and synchrotron self-absorption. The observed hard spectra suggest heavy pairloading in GRBs. The re-emission of the generated pairs results in the energy transmission from high-energy gamma-rays to long-wavelength radiation. Due to strong self-absorption, the synchrotron radiation by pairs is in optically thick regime, showing a thermal-like spectral bump in the extreme-ultraviolet/soft Xray band, other than the peak from the main burst. Recently, the prompt soft X-ray emission of GRB 031203 was detected thanks to the discovery of a delayed dust echo, and it seems to be consistent with the model prediction of a doublepeak structure. The confirmation of the thermal-like feature and the double-peak structure by observation would indicate that the dominant radiation mechanism in GRBs is synchrotron rather than inverse-Compton radiation.
\end{abstract}

Subject headings: gamma-rays: bursts — radiation mechanisms: nonthermal — relativity

\section{Introduction}

In the past few years, a standard model was well established in which the gamma-ray burst (GRB) afterglows result from the relativistic blast-waves sweeping up the ambient medium of GRBs (Mészáros 2002). However, the prompt emission of GRBs is believed to be irrelevant to ambient medium, and its radiation mechanism is still poorly known so far.

The recent definite proof of GRB 030329 associated with a type Ib/c supernova confirmed, as long suspected, that GRBs, at less the long class, originate from explosions of massive stars in distant galaxies (Stanek et al. 2003; Hjorth et al. 2003). Since GRBs are events occurring on stars, the emission region may be compact, and the huge energy release

\footnotetext{
${ }^{1}$ Particle Astrophysics Center, Institute of High Energy Physics, Chinese Academy of Sciences, Beijing 100039, China
} 
will lead to the formation of $e^{ \pm}, \gamma$ fireballs, exhibiting thermal-like spectra. But the GRB spectra are non-thermal and hard, with a significant fraction of the energy above the $e^{ \pm}$pair formation energy threshold. For a photon with tens of $\mathrm{MeV}$ to escape freely, avoiding $\gamma \gamma$ interactions, the fireball must be ultra-relativistic expanding, with $\Gamma \gtrsim 100$ (Lithwick \& Sari 2001, and references therein). The afterglow studies has also confirmed the presence of ultra-relativistic motion. However, if the intrinsic emission, before leaking out from fireball, includes radiation with even higher energy, say, beyond $\mathrm{GeV}$, these radiation still suffers $\gamma \gamma$ absorption, leading to pair loading in GRBs. In context of relativistic fireball model, Li et al. (2003; Li03 hereafter) found that, in a wide range of model parameters, the resulting pairs may dominate those electrons associated with fireball baryons. The presence of abundant pairs would affect the behaviors of the early afterglow from reverse shocks (Li03), and may also emit particular signals in the bursting phase.

We discuss in this Letter the prompt GRB emission, with emphasis on the re-emission by the secondary $e^{ \pm}$pairs. If the energy density in the emission region is dominated by magnetic field, the pairs would re-emit mainly by synchrotron radiation, rather than IC process (e.g., Pilla \& Loeb 1998). Due to strong self-absorption, the pair emission appears as a thermal-like bump in the GRB spectrum, similar to the feature discussed by Kobayashi, Mészáros \& Zhang (2004) in the context of reverse shock emission. (Fan \& Wei 2004 have also studied the pair emission, but with less stress on the self-absorption effect.) We further show that the intense soft X-ray emission in GRB 031203, inferred by the delayed dust halo (Vaughan et al. 2004 [V04]; Watson et al. 2004 [W04]), can be accounted for by the spectral bump due to pair-loading. This is of significant interest, since this feature could give a diagnostics for the magnetic field in the fireball (Kobayashi, Mészáros \& Zhang 2004) and the dominant radiation mechanism in GRBs.

\section{Pair loading in GRB fireballs}

Consider a GRB central engine that produce a relativistic wind outflow, with an isotropic energy $E$, a bulk Lorentz factor $\Gamma$ and a width $\Delta$. The energy carried in the outflow may be composed of two components, the bulk kinetic energy of baryons $\left(E_{k}\right)$ and the energy of magnetic field $\left(E_{B}\right)$. The ratio between them can be defined as $\sigma \equiv E_{B} / E_{k}$ (e.g., Zhang \& Mészáros 2002). These energies are carried from the central engine to some radius $R$ where GRB emission arises. As in Li03, the emission site can be constrained by the non-thermal spectra and rapid varying light curves of GRBs, leading to typical value of $R \sim 10^{14} \mathrm{~cm}$ for

$\Gamma \sim 300$. The width of outflow is $\Delta \lesssim 10^{12} \mathrm{~cm}$ for a wind lasting a duration of $T \lesssim 100 \mathrm{~s}$. Thus the emission region can be regarded as a thin shell with $\Delta \ll R$. 
In the kinetic-energy dominated model $(\sigma<1)$, the bulk kinetic energy is dissipated by internal shock waves within the unsteady outflow, where the magnetic field strength $B$ is in the equipartition value $B \sim\left(8 \pi U_{\gamma}\right)^{1 / 2}=10^{4} L_{\gamma, 51}^{1 / 2} \Gamma_{300}^{-1} R_{14}^{-1} \mathrm{G}$, with $L_{\gamma}=10^{51} L_{\gamma, 51} \mathrm{erg} \mathrm{s}^{-1}$ the GRB luminosity, $\Gamma_{300}=\Gamma / 300$ and $R_{14}=R / 10^{14} \mathrm{~cm}$. In the magnetic-energy dominated model, the magnetic field could be stronger than the equipartition value, leading to a small radiation-to-magnetic energy ratio in emission region, $Y \equiv U_{\gamma} / U_{B}<1$. Though broad band fits of afterglows generally give $Y>1$ for shocked-medium, the presence of highly magnetized ejecta is suggested by some recent works (e.g., Zhang, Kobayashi, \& Mészáros 2003). Here we will assume $Y<1$ for GRBs, and scale the magnetic field as $B=10^{4} B_{4} \mathrm{G}$.

Due to the large luminosity and hard spectrum of a GRB, intrinsic high energy gammarays produced in the GRB emission region could be absorbed for pair production. As in Li03, the cut-off energy, above which the photons suffer strong absorption, and the number of produced pairs can be estimated from the observed GRB spectra. The observed photon spectra of GRBs can be approximated by a broken power-law, with a high-energy portion of the form $d N_{\gamma} / d \epsilon \propto \epsilon^{-\beta}$ for $\epsilon>\epsilon_{p}$, where $\epsilon_{p} \sim m_{e} c^{2}$ is the energy at the broken point and the index $\beta \sim 2-3$. The number of the produced secondary pairs is equal to the absorbed photons above $\epsilon_{c u t}$. Assuming the intrinsic spectrum above $\epsilon_{c u t}$ follows the same power law below $\epsilon_{\text {cut }}$, we calculate the pair number as $N_{ \pm}=N_{\gamma}\left(>\epsilon_{c u t}\right) \simeq\left(E_{\gamma} / \epsilon_{p}\right)\left(\epsilon_{c u t} / \epsilon_{p}\right)^{-(\beta-1)}$. Since the timescale of $\gamma \gamma$ collisions (comoving frame), $t_{\gamma \gamma}^{\prime} \simeq\left[\left(\sigma_{T} / 5\right) n_{\gamma}^{\prime} c\right]^{-1}=0.2 \Gamma_{300} R_{14}^{2} L_{\gamma, 51}^{-1}$, is usually shorter than the dynamical time (comoving frame), $t_{d y n}^{\prime} \simeq R / \Gamma c=10 R_{14} \Gamma_{300}^{-1} \mathrm{~s}$, the resulting pairs remain inside the outflow.

As in Li03, $\epsilon_{\text {cut }}$ should be defined by the photon energy at which the optical depth for $\gamma \gamma$ absorption equals unity, $\tau_{\gamma \gamma}(\epsilon)=1$, where the optical depth can be given by a simplified expression under the thin-shell assumption of the emission region, $\tau_{\gamma \gamma}(\epsilon)=(11 / 180) \sigma_{T} N_{\gamma}(>$ $\epsilon) / 4 \pi R^{2}$ (Lithwick \& Sari 2001). Furthermore, the observed cut-off energy must be larger than $\Gamma m_{e} c^{2}$. In summary,

$$
\epsilon_{\text {cut }}=\max \left[0.3\left(\frac{R_{14}^{2}}{E_{\gamma, 52} \epsilon_{0}^{\beta-2}}\right)^{1 /(\beta-1)} \Gamma_{300}^{2} ; 0.2 \Gamma_{300}\right] \mathrm{GeV}
$$

where $\epsilon_{0}=\epsilon_{p} / m_{e} c^{2}$, and hereinafter the numerical coefficient corresponds to $\beta=2.4$. It can be seen that the detection of the cut-off energy can help to constrain $\Gamma$ and $R$. EGRET had detected prompt GeV emission, without obvious attenuation, in several GRBs (e.g., GRB 930131; Sommer et al. 1994). We expect that the future satellite GLAST, which works in 10 $\mathrm{MeV}-300 \mathrm{GeV}$ range, could observe such a cut off at multi-GeV. ${ }^{1}$ With $\epsilon_{\mathrm{GeV}}=\epsilon_{\text {cut }} / 1 \mathrm{GeV}$,

\footnotetext{
${ }^{1}$ Cosmic infrared background can also absorb high energy photons, but primarily in TeV range (Salamon
} 
the pair number is written as

$$
N_{ \pm} \simeq 3 \times 10^{53} E_{\gamma, 52} \epsilon_{\mathrm{GeV}}^{-(\beta-1)} \epsilon_{0}^{\beta-2}
$$

For comparison, the number of baryonic electrons in the fireball is $N_{b}=E /(1+\sigma) \Gamma m_{p} c^{2}=$ $2 \times 10^{52} E_{52} \Gamma_{300}^{-1}(1+\sigma)^{-1}$, with $E_{52}=E / 10^{52} \mathrm{erg}$. So pairs become the dominant component. The baryonic electrons are expected to be responsible for the prompt hard X-ray emission, whilst the pairs might give rise to low energy emission, discussed below.

\section{Extreme-ultraviolet bump in the prompt emission}

Since the energy of a generated $e^{+}\left(e^{-}\right)$comes primarily from the photon with lager energy between the two colliding ones, the initial energy distribution of the generated pairs would follow the form of the high-energy spectral tail, i.e., $d n_{ \pm} / d \gamma_{e} \propto \gamma_{e}^{-\beta}$ for $\gamma_{e}>\gamma_{ \pm}$, where $\gamma_{ \pm}$corresponds to the cut-off energy, $\gamma_{ \pm}=\epsilon_{\text {cut }} / 2 \Gamma m_{e} c^{2} \simeq 3.3 \epsilon_{\mathrm{GeV}} \Gamma_{300}^{-1}$. These pairs will cool down by synchrotron rather than IC radiation in the $Y \lesssim 1$ condition here. Because of the strong magnetic field in the emission region, the synchrotron-cooling timescale of pairs, $t_{\text {syn }}^{\prime}=8 B_{4}^{-2} \gamma_{ \pm}^{-1} \mathrm{~s}$, is shorter than the fireball dynamical time, implying that the pairs are always fast cooling. We assume that pair annihilation is negligible, confirmed later.

For these fast cooling pairs, their energies are emitted quickly. As a result, the energy above $\epsilon_{c u t}$ in the intrinsic spectrum re-arises as the pair emission. The luminosity of the pair emission is given by

$$
L_{ \pm} \simeq \frac{\beta-1}{\beta-2} \frac{N_{ \pm} \epsilon_{c u t}}{T} \simeq 2 \times 10^{50} L_{\gamma, 51}\left(\frac{\epsilon_{0}}{\epsilon_{\mathrm{GeV}}}\right)^{\beta-2} \mathrm{erg} \mathrm{s}^{-1}
$$

with $L_{\gamma}=E_{\gamma} / T=10^{51} L_{\gamma, 51} \mathrm{erg} \mathrm{s}^{-1}$ the GRB luminosity, and the characteristic synchrotron frequency is

$$
\nu_{ \pm}=0.9 \times 10^{14} \Gamma_{300}^{-1} \epsilon_{\mathrm{GeV}}^{2} B_{4} \mathrm{~Hz} .
$$

If we assume $Y \ll 1$, the synchrotron radiation plays a dominant role of pair cooling, rather than IC process. In this condition, the luminosity $L_{ \pm}$will peak at frequency $\nu_{ \pm}$. Thus a very intense optical flash will emerge accompanying the prompt gamma-rays if neglecting the self-absorption. However, as shown in the following, the self-absorption is strong in such low energy range, with the absorption frequency $\nu_{a} \gg \nu_{ \pm}$, i.e., most of the pair emission occurs in the optically thick regime. Similar to the case of reverse flash in the condition of

\& Stecker 1998). 
$\nu_{c}<\nu_{m}<\nu_{a}$, which is discussed by Kobayashi et al. (2004), a thermal-like bump will arise in the low energy range of a GRB spectrum.

The self-absorption suppresses the emission below absorption frequency $\nu_{a}$, and the suppressed emission energy is redistributed again among the pairs, preventing the pairs cool down immediately. So, the pairs and the radiation obtain a mechanism to exchange their energies. The final result is that the initial injected pair energy is redistributed among pairs and radiation, leading to a bump in the spectrum. The emission in the hard X-ray band is not in the optically thick regime, and is not involved in the energy redistribution. In the GRB duration $T$, the pair energy is radiated around $\nu_{a}$, where the flux is given by $F_{\nu_{a}} \simeq L_{ \pm} / 4 \pi D_{L}^{2} \nu_{a}$, with $D_{L}$ the GRB luminosity distance. We follow the simple way by Sari \& Piran (1999)to estimate the maximal flux as a blackbody with the pair temperature, $F_{\nu_{a}, b b} \approx \pi\left(R_{\perp} / D_{L}\right)^{2}\left(2 \nu_{a}^{2} / c^{2}\right) k T_{ \pm}$, where $R_{\perp} \simeq R / \Gamma$ is the observed size of the fireball, the pair temperature is $k T_{ \pm} \simeq \Gamma \gamma_{a} m_{e} c^{2} / 3$, and $\gamma_{a}$ is the pair Lorentz factor that corresponds to $\nu_{a}$ and is given by $\left(2 \pi m_{e} c \nu_{a} / \Gamma e B\right)^{1 / 2}$. Equating $F_{\nu_{a}, b b} \simeq F_{\nu_{a}}$ yields the self-absorption frequency

$$
\nu_{a} \simeq 1 \times 10^{16} L_{ \pm, 50}^{2 / 7} \Gamma_{300}^{3 / 7} R_{14}^{-4 / 7} B_{4}^{1 / 7} \mathrm{~Hz}
$$

which is in the extreme-ultraviolet (EUV) band. Since $\nu_{a} \gg \nu_{ \pm}$, most of the emission is absorbed and re-distributed, giving rise to a black-body like bump in the GRB spectrum, with peak frequency around $\nu_{a}$ (eq.[5]) and luminosity $L_{ \pm}$(eq.[3]).

The comoving-frame annihilation timescale of the pairs in the thermal-like bump, with the comoving-frame temperature $\gamma_{a} m_{e} c^{2} / 3$, is $t_{a n n}^{\prime} \simeq\left(\sigma_{a n n} n_{ \pm} c\right)^{-1}$, where $\sigma_{a n n} \simeq\left(3 \sigma_{T} / 8 \gamma_{a}\right)\left(\ln 2 \gamma_{a}-\right.$ $1)$ is the annihilation cross section, and the pair number density is given by $n_{ \pm} \approx n_{\gamma}(>$ $\left.\epsilon_{\text {cut }}\right) \simeq L_{ \pm} / 4 \pi R^{2} \Gamma c \epsilon_{\text {cut }}$. Note the baryonic electrons are neglected. The annihilation fraction of pairs, $f_{a n n}$, can be estimated by $t_{\text {ann }}^{\prime-1}$ times the comoving-frame dynamical timescale $t_{d y n}^{\prime}$, $f_{\text {ann }} \sim 0.08\left(L_{ \pm, 51} / \Gamma_{300}^{3} R_{14}\right)\left(\gamma_{ \pm} \gamma_{a} / 10\right)^{-1}$. Since $f_{\text {ann }} \ll 1$, the annihilation in the bump is really negligible.

Notice that $\nu_{a}$ is insensitive to all the parameters (eq. 5), and would be fixed in the EUV range for various GRBs. However, as it propagates, the EUV radiation from a GRB is subject to intergalactic or galactic absorption (e.g., Gou et al. 2004), hence only the optical/UV or soft X-ray emission is expected for observation. Below $\nu_{a}$ the spectrum behaves as $F_{\nu<\nu_{a}}=F_{\nu_{a}}\left(\nu / \nu_{a}\right)^{2}$, then the observed pair emission at $1 \mathrm{eV}$ is

$$
F_{\nu}^{\mathrm{ob}}(1 \mathrm{eV}) \simeq 0.9 \frac{L_{\gamma, 51}(1+z)^{3}}{D_{28}^{2} \nu_{a, 16}^{3}}\left(\frac{\epsilon_{0}}{\epsilon_{\mathrm{GeV}}}\right)^{\beta-2} \mathrm{mJy}
$$

where $\nu_{a, 16}=\nu_{a} / 10^{16} \mathrm{~Hz}$, and we have obviously shown the dependence on redshift $z$. Whereas in the band above $\nu_{a}$, the emission is in optically thin regime and still exhibits the 
form radiated by the initial pairs, $F_{\nu>\nu_{a}}=F_{\nu_{ \pm}}\left(\nu / \nu_{ \pm}\right)^{-\beta / 2}$, where $F_{\nu_{ \pm}} \simeq L_{ \pm} / 4 \pi D_{L}^{2} \nu_{ \pm}$. If observed at $1 \mathrm{keV}$, the flux contributed by pairs is then

$$
\begin{aligned}
F_{\nu}^{\mathrm{ob}}(1 \mathrm{keV}) \simeq 3 \times 10^{-8} \frac{L_{\gamma, 51}}{D_{28}^{2}}\left[\frac{\epsilon_{0}^{2} B_{4}}{\Gamma_{300}(1+z)}\right]^{(\beta-2) / 2} \\
\\
\quad \mathrm{erg} \mathrm{cm}^{-2} \mathrm{~s}^{-1} \mathrm{keV}^{-1} .
\end{aligned}
$$

This calculation is valid until at high enough frequency where the main GRB peak dominates the emission. For the typical parameters, the contrast of the thermal bump relative to the power-law spectrum $\left(\nu_{a} / \nu_{ \pm}\right)^{\beta / 2-1}$ (Fig. 1) is a factor of a few, and the bump is weak. For larger index $\beta \sim 3$ the contrast is larger, and though the energy injected into the pairs becomes smaller, the thermal bump still sticks out above the primary emission component. The prompt optical/UV (eq.6) and X-ray (eq.7) emission are expected to be observed by the UVOT and XRT detector, respectively, on board the up-coming Swift satellite.

As a result, the intrinsic high-energy emission in the GRB spectrum is absorbed and then transferred to a thermal-like bump in the EUV band, as shown in Fig. 1. Since these features are expected to arise if $Y \ll 1$, the observation would provide a constraint on the magnetization parameter and the radiation mechanism in GRBs.

\section{GRB 031203}

GRB 031203 was detected by INTEGRAL as a single-pulse burst with a duration of $30 \mathrm{~s}$ and a peak flux of $1.3 \times 10^{-7} \mathrm{erg} \mathrm{s}^{-1} \mathrm{~cm}^{-2}$ in the $20-200 \mathrm{keV}$ band (Götz et al. 2003; Mereghetti \& Götz 2003). A double exponential approximation to the single-pulse light curve yields an estimated fluence of $4 \times 10^{-7} \mathrm{erg} \mathrm{cm}^{-2}(20-200 \mathrm{keV})$ (Prochaska et al. 2004; P04). With the redshift $z=0.1055$ measured from the optical observation of the host galaxy, the $k$-correction isotropic-equivalent energy release is estimated to be $E_{\text {iso }}(20-2000 \mathrm{keV})=2.6 \times 10^{49} \mathrm{erg}$ $\left(h=0.7, \Omega_{\Lambda}=0.7\right.$ and $\left.\Omega_{m}=0.3\right)(\mathrm{P} 04)$.

6 hours after the burst, XMM-Newton discovers a time-dependent, dust-scattered Xray halo around the burst (V04; W04). The halo brightness implies an initial soft X-ray pulse consistent with the burst. Since few satellites observe GRBs in soft X-ray band, this observation is important for us to know the emission features of GRBs in such low energy range. Based on the hypothesis of the column of dust along the sightline toward GRB 031203, V04 and W04 estimates a source flux of $1.5 \pm 0.8 \times 10^{-7} \mathrm{erg} \mathrm{cm}^{-2} \mathrm{~s}^{-1}$ with photon index $\beta_{X}=2.2 \pm 0.3$ in the $0.2-10 \mathrm{keV}$ band. This leads them to regard GRB 031203 as an $\mathrm{X}$-ray flash with peak energy at $\lesssim 10 \mathrm{keV}$. However, P04 argue that the above analysis has overestimated the scattering column of dust, which may be 4.4 (or even 27) times larger, 
and hence the source should be 4.4 (27) times smaller in flux. Thus, we take in the following discussion the source flux of the dust halo as $3.4 \pm 1.8(0.56 \pm 0.30) \times 10^{-8} \mathrm{erg} \mathrm{cm}^{-2} \mathrm{~s}^{-1}$ in the $0.2-10 \mathrm{keV}$ band, which corresponds to a fluence of $10 \pm 5.4(1.7 \pm 0.9) \times 10^{-7} \mathrm{erg} \mathrm{cm}^{-2}$ for a duration of $30 \mathrm{~s}$.

The observational results of GRB 031203 is consistent with the double-peak spectral shape described in $\S 3$. This rests on two points. First, the photon index of $\sim 2.2$ in $0.2-10$ $\mathrm{keV}$ band implies a soft X-ray peak, with most energy released at $\lesssim 0.2 \mathrm{keV}$. And second, the fluence in $20-200 \mathrm{keV}$ band is larger than that extrapolated from the $0.2-10 \mathrm{keV}$ band using the photon index $\beta_{X} \sim 2.2$, suggesting a second peak in the hard X-ray band. The observed hard X-ray emission is only comparable to or even slightly smaller than the soft X-ray one in fluence, but we expect that, since the redshift of GRB 031203 is low and hence the peak energy is less redshifted, the hard X-ray emission could peak at higher energy, $\epsilon_{p}>200 \mathrm{keV}$. If peaking at $\sim 1 \mathrm{MeV}$, the hard X-ray fluence would be larger by a factor of $\sim 5$, and dominates the soft one. It is unfortunate that there is not reliable time-integrated spectrum of GRB 031203 above $20 \mathrm{keV}$ that is given. Therefore we cannot compare the two peaks for more details. Since the soft X-ray spectrum of GRB 031203 follows a power law, the thermal-like bump should be at an energy below the observation window, $\lesssim 0.2 \mathrm{keV}$, consistent with predicted in eq.(5).

There may be some caveats here. One may think that a double-peak structure can also be interpreted as synchrotron self-Compton radiation provided $Y>1$. But in this condition, the pairs from $\gamma \gamma$ production will loss energy by IC radiation and result in a spectral bump at $\gamma_{ \pm}^{2} \epsilon_{p} \lesssim 100 \mathrm{MeV}$, something conflicting with the observation of GRBs in high energy band (Schaefer et al. 1998). A further difficulty for SSC to interpret the double peaks is that there may be many Compton order if $Y>1$, and each higher Compton order will dominate over the previous one by the same amount $Y$ until the typical emitted energy reaches the electron energy. Only a small fraction of the radiated power would therefore be observed in the sub-MeV band (e.g., Ghisellini et al. 2000), resulting in energy crisis of GRBs.

One may also think that the source of the dust halo is the X-ray afterglow in early time rather than the prompt burst. We believe this can be ruled out. The dust halo consists of two narrow rings, and V04 have interpret them as being due to the prompt burst scattered by two distinct scattering screens. One may still propose that only a single scattering screen is required as long as there is a complex early time structure of the X-ray afterglow. However, if so the later ring should evolve with a certain time delay with respect to the first one, which is in contrast with the observation (see $\theta-t$ curves in fig. 3 of V04). The ring is rather narrow, implying that the intrinsic emission is a short pulse, as opposed to the smooth afterglow behavior. In fact, since the ring is narrow, $\Delta \theta \sim 20 \operatorname{arcsec}$ in angular width, the 
pulse duration is limited to $\Delta t \lesssim D \Delta \theta^{2} / c \sim 800 \mathrm{~s}$, where the distances of the screens from earth are $D \sim 1 \mathrm{kpc}$ for both rings (V04). Because of causality, the dynamical time is also limited to $t \sim \Delta t \lesssim 800 \mathrm{~s}$.

\section{Summary and discussion}

We have studied the prompt GRB emission, allowing for $\gamma \gamma$ pair production and synchrotron self-absorption. Inferred by the observed characteristics of GRB emission, the resulting pairs usually dominate the baryonic electrons. The pairs will give rise to further emission by synchrotron radiation if in the strong magnetic field, which is also responsible to the prompt sub-MeV emission. However, due to strong self-absorption the pair emission exhibits a thermal-like bump in the extreme UV/soft X-ray band, other than the peak in the hard X-ray band. Since the feature emerges for $Y<1$, its observation gives a diagnostics for the magnetic energy density in the fireball (Kobayashi, Mészáros , \& Zhang 2004). The recent observation of a dust halo around GRB 031203 infers a spectral peak of the prompt burst emission in the soft X-ray band, which seems to be consistent with the predicted double-peak structure.

Some primary hypotheses have been taken in our calculation. First, we assume that the emission region is transparent for Compton scattering, even though the secondary pairs increase significantly the total optical depth. For typical parameter values this assumption is protected. However, in some extreme cases with quite small $\Gamma$ and $R$, the secondary pairs may form an optically thick screen again (Guetta, Spada \& Waxman 2001; Kobayashi, Ryde \& MacFadyen 2002), which degrades the gamma-rays and results in an X-ray flash (XRF; Mészáros et al. 2002). If so, our calculation using eq. (2) may underestimate the pair-loading in XRFs, which may need detailed works of numerical simulation (e.g., Pe'er \& Waxman 2003). Secondly, we assume strong magnetic field, $Y<1$, in the emission region. If $Y>1$, the pairs lose most energy by IC scattering the GRB photons, and the IC photons are not self-absorbed again since beyond the optically thick regime, hence no effective energy exchange between pairs and photons is established and the bump disappears. Therefore, once UV/soft X-ray bumps are detected this will infer $Y<1$ and that it is synchrotron rather than IC radiation that gives rise to the sub-MeV emission of GRBs.

This work was supported by the National 973 Project and the Special Funds for Major State Basic Research Projects. 


\section{REFERENCES}

Fan, Y. Z., \& Wei, D. M. 2004, MNRAS in press (astro-ph/0403163)

Ghisellini, G., Celotti, A., \& Lazzati, D. 2000, MNRAS, 313, L1

Götz, D., Mereghetti, S., Beck, M., Borkowski, J., Mowlavi, N. 2003, GCN 2459

Gou, L. J., Mészáros , P., Abel, T., \& Zhang, B. 2004, ApJ, 604, 508

Guetta, D., Spada, M., \& Waxman, E. 2001, ApJ, 557, 399

Hjorth, J., et al. 2003, Nature, 423, 847

Kobayashi, S., Mészáros , P., \& Zhang, B. 2004, ApJ, 601, L13

Kobayashi, S., Ryde, F., \& MacFadyen, A. 2002, ApJ, 577, 302

Li, Z., Dai, Z. G., Lu, T., \& Song, L. M. 2003, ApJ, 599, 380 (Li03)

Lithwick, Y., \& Sari, R. 2001, ApJ, 555, 540

Mereghetti, S., \& Götz, D. 2003, GCN 2460

Mészáros , P. 2002, ARA\&A, 40, 137

Mészáros , P., Ramirez-Ruiz, E., Rees, M. J., \& Zhang, B., 2002, ApJ, 578, 812

Pe'er, A., \& Waxman, E., 2003, ApJ submitted (astro-ph/0311252)

Pilla, R., \& Loeb, A., 1998, ApJ, 494, L167

Prochaska, J., et al. 2004, astro-ph/0402085 (P04)

Salamon, M. H., \& Stecker, F. W. 1998, ApJ, 493, 547

Sari, R., \& Piran, T. 1999, ApJ, 520, 641

Schaefer, B. E., et al. 1998, ApJ, 492, 696

Sommer, M., et al. 1994, ApJ, 422, L63

Stanek, K. Z., et al. 2003, ApJ, 591, L17

Vaughan, S., et al. 2004, ApJ, 603, L5 (V04)

Watson, D., et al. 2004, ApJ, 605, L101 (W04) 
Zhang, B., Kobayashi, S, \& Mészáros , P. 2003, ApJ, 595, 950

Zhang, B., \& Mészáros , P. 2002, ApJ, 581, 1236 


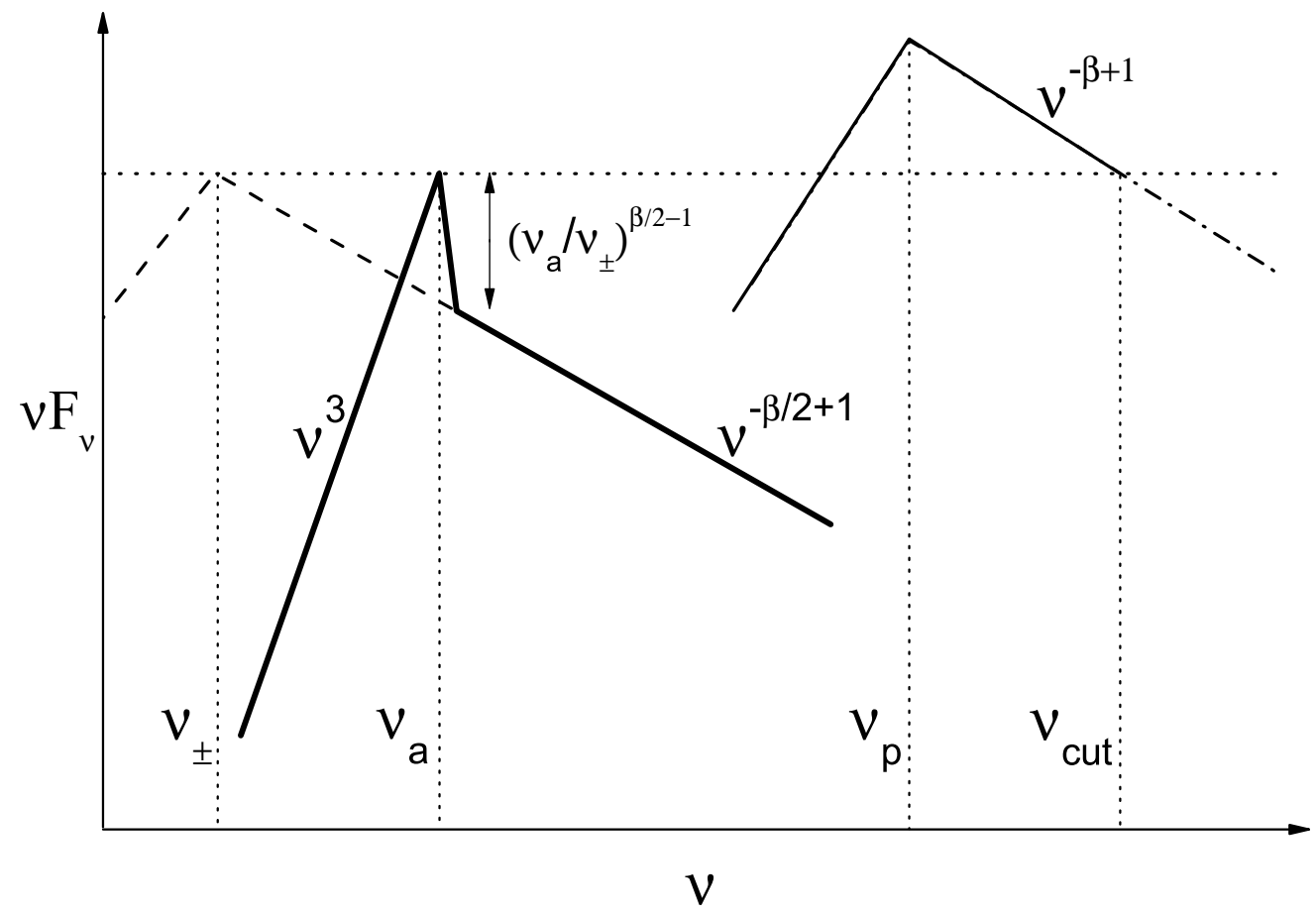

Fig. 1. - The schematic diagram of the $\nu F_{\nu}$ spectrum of the GRB prompt emission. Note that $\nu_{p}=\epsilon_{p} / h$ and $\nu_{c u t}=\epsilon_{c u t} / h$. The thin solid line shows the GRB emission with cut-off above $\nu_{c u t}$ due to $\gamma \gamma$ pair production, while the dashed-dotted line is the intrinsic spectrum without cut-off. The synchrotron radiation by the resulting pairs is shown by the thick solid or dashed lines, corresponding to with or without self-absorption, respectively. The maximum around $\nu_{a}$ would appear as a thermal-like peak. 